\title{
Optimal Allocation of Generalized Power Sources in Distribution Network Based on Multi-Objective Particle Swarm Optimization Algorithm
}

\author{
Li Ran ${ }^{1}$, Yuan Jingwei ${ }^{2}$, Song Yundong ${ }^{2}$, Yang Yingxuan ${ }^{2}$ and Meng Tao ${ }^{3}$ \\ ${ }^{1}$ Shenyang Power Supply Company, Liaoning Electric Power Company Limited, Shenyang 110006, China \\ ${ }^{2}$ Electric Power Research institute Liaoning Electric Power Company Limited, Shenyang 110006, China \\ ${ }^{3}$ Electric Power Research institute of Jilin Electric Power Co. of State Grid, Changchun 130021, China
}

\begin{abstract}
Optimal allocation of generalized power sources in distribution network is researched. A simple index of voltage stability is put forward. Considering the investment and operation benefit, the stability of voltage and the pollution emissions of generalized power sources in distribution network, a multi-objective optimization planning model is established. A multi-objective particle swarm optimization algorithm is proposed to solve the optimal model. In order to improve the global search ability, the strategies of fast non-dominated sorting, elitism and crowding distance are adopted in this algorithm. Finally, tested the model and algorithm by IEEE-33 node system to find the best configuration of GP, the computed result shows that with the generalized power reasonable access to the active distribution network, the investment benefit and the voltage stability of the system is improved, and the proposed algorithm has better global search capability.
\end{abstract}

\section{Introduction}

Large scale of distributed generators (DGs) supply will increase the complexity and uncertainty of planning of distribution network (DN), and even has a significant effect to this system, such as the quality of voltage deteriorate, the reliability of power supply reducing [1]. Improper allocation of DGs in active distribution network has been studied. In [2], considering the timing characteristics of the side of source and load, a mathematical model of network loss and power loss goal is established and an improved genetic algorithm is proposed to solve this model. In [3], an economic evaluation index of investment, operation, fuel and exhaust emission is set up to study DGs programming problem under different scenarios. Different methods were proposed to evaluating the planning effect of DGs in [4], however, in this methods, the multi-objective optimization problem is transformed into a single objective problem, and the choice of weighted coefficient directly affects the results of optimization, which make the considered factors of allocation schemes not comprehensive.

The concept of generalized power (GP) refers to a device that emits a certain active or reactive power, mainly DG and capacitor group. The existing research mainly puts DG and capacitor group separately and rarely considers the optimum allocation problem of GP. In [5], a multi-objective optimization model is put forward to study the optimal allocation of DG under different conditions by using the multi-objective particle swarm algorithm. The non-dominated sorting genetic algorithm is used to optimize capacitors in distribution network in [6]. At present, few literatures consider the problem of optimal allocation of GP in ADN. Therefore, optimal allocation of GP has an important significance of operation of ADN.

In view of above problems, this paper puts forward the index of voltage stability based on simple network flow. From the point of view of economic benefit of investment operation, voltage stability and pollution emissions, a multi-objective mathematical model considering GP allocation is established. A multiobjective particle swarm optimization algorithm is proposed in this paper. Finally, tested the model and algorithm by IEEE-33 node system, and study the situation of economic benefits, voltage stability and emissions under different load level to determine the best configuration of GP.

\section{Multi-objective optimization model}

\subsection{The benefits of investment and operation}

Comprehensive consideration of investment, operation cost and the marginal benefit during the process of planning, and the corresponding mathematic models for $f_{1}$ :

$$
f_{1}=B_{\mathrm{M}}+B_{\mathrm{GP}}-C_{\mathrm{GP}}+\sum_{i=1}^{2} B R_{i}
$$


where, $B_{\mathrm{M}}, B_{\mathrm{GP}}, C_{\mathrm{GP}}$ denote power selling benefit of microgrid, power generation benefit of GP, installation cost of GP, respectively. $B R$ is the marginal benefit of installing GP, where, BR1 and BR2 denote the benefit of delay grid upgrade and the benefit of improve network loss, respectively.

\subsection{Voltage stability index}

Considering the operation of voltage stability of power system, and the corresponding mathematic models for $f_{2}$ :

$$
f_{2}=V S I=\frac{1}{N} \sum_{i=1}^{N}\left(2 V_{i+1}-V_{i}\right)
$$

where $V_{i}$ and $V_{i+1}$ denote voltage of first node and voltage of lend node. The parameter $i$ is referred to as the node number. $N$ is the total number of nodes.

From (2), the voltage stability index is only concerned with voltage amplitude, and when the voltage of end node down to the half of the first node, the phenomenon of voltage instability could occur in system.

\subsection{Pollution emissions index}

Considering the emission index of $\mathrm{CO}_{2}, \mathrm{NO}_{x}, \mathrm{SO}_{2}, \mathrm{CO}$ and other pollution gas, the corresponding mathematic model for $f_{3}$ :

$$
f_{3}=\sum_{i=1}^{N} \sum_{j=1}^{N_{\mathrm{GP}}} \sum_{k=1}^{N_{\mathrm{g}}} S_{\mathrm{GP}}(i j) \times w_{k} \times E R_{j k} \times \alpha_{j} \times 8760
$$

where, $N_{\mathrm{GP}}$ and $N_{\mathrm{g}}$ denote the type of GP and the type of pollution emission, respectively. $S_{\mathrm{GP}}(i j)$ is the output power of GP from the type of $j$ at the node of $i . w_{k}$ is the weight coefficient of the pollution emission type of $k . E R_{j k}$ is the pollution emission rate of GP. $\alpha_{j}$ is the coefficient of carbon emissions.

\subsection{Configuration optimization mathematical model of GP}

Considering the index of benefit of GP investment, voltage stability and pollution emission, the corresponding multi objective optimization mathematical moder as for:

$$
\left\{\begin{array}{l}
F(X)=\left\{f_{1}(X), f_{2}(X), f_{3}(X)\right\} \\
\text { s.t. } g(X) \leq 0, \quad h(X)=0
\end{array}\right.
$$

where, $g(X)$ is the constraints of inequality, $h(X)$ is the constraints of equality, and the parameter $X$ is a collection of GP's capacity and location and other variables.

Current equation constraints are mainly constraint:

$$
\left\{\begin{array}{l}
P_{\mathrm{GP}_{-} i}-P_{\mathrm{L}_{-} i}=V_{i} \sum_{j=1}^{N} V_{j}\left(G_{i j} \cos \delta_{i j}+B_{i j} \sin \delta_{i j}\right) \\
Q_{\mathrm{GP}_{-} i}-Q_{\mathrm{L}_{-} i}=V_{i} \sum_{j=1}^{N} V_{j}\left(G_{i j} \sin \delta_{i j}+B_{i j} \cos \delta_{i j}\right)
\end{array}\right.
$$

where, $P_{G P_{-} i}$ and $Q_{G P_{-} i}$ denote active power and reactive power from GP at node i, respectively. $P_{L_{-} i}$ and $Q_{L_{-} i}$ denote active power and reactive power from load at node i, respectively. $G_{i j}, B_{i j}$ and $\delta_{i j}$ denote admittance and voltage phase angle parameters, respectively.

Inequality constraint conditions including the installed capacity of GP, the limiting value of node voltage and branch current:

$$
\left\{\begin{array}{l}
S_{\mathrm{GP}_{-} j} \leq S_{\mathrm{GP}_{j} j}^{\max }, i \in N \\
U_{i}^{\min } \leq U_{i} \leq U_{i}^{\max } \\
I_{i j} \leq I_{i j}^{\max }
\end{array}\right.
$$

\section{MPSO algorithm}

In this paper, the multi-objective particle swarm (MPSO) algorithm is proposed based on two order particle swarm algorithm. In view of the existence problem of premature and local search on traditional algorithm, the method of linear increasing weight is used to change the weight coefficient. The formula of speed and location of MPSO as follow:

$$
\begin{gathered}
v_{i k}(t+1)=\omega v_{i k}(t)+c_{1} r_{1}\left[p_{i k}(t)-2 x_{i k}(t)+x_{i k}(t-1)\right]+ \\
c_{2} r_{2}\left(g_{i k}(t)-2 x_{i k}(t)+x_{i k}(t-1)\right) \\
x_{i k}(t+1)=x_{i k}(t)+v_{i k}(t+1)
\end{gathered}
$$

where, $v_{i k}(t)$ and $x_{i k}(t)$ denote particle speed and position, respectively. $p_{i k}(t)$ is the individual extreme position of particle $i$ in the $t$ iteration under $k$ dimension. $g_{i k}(t)$ is the global extreme position of particle $i . \omega$ is the inertia weight, and $c_{1}, c_{2}$ is the factor of learning. $r_{1}$ and $r_{2}$ is the random number under $[0,1]$.

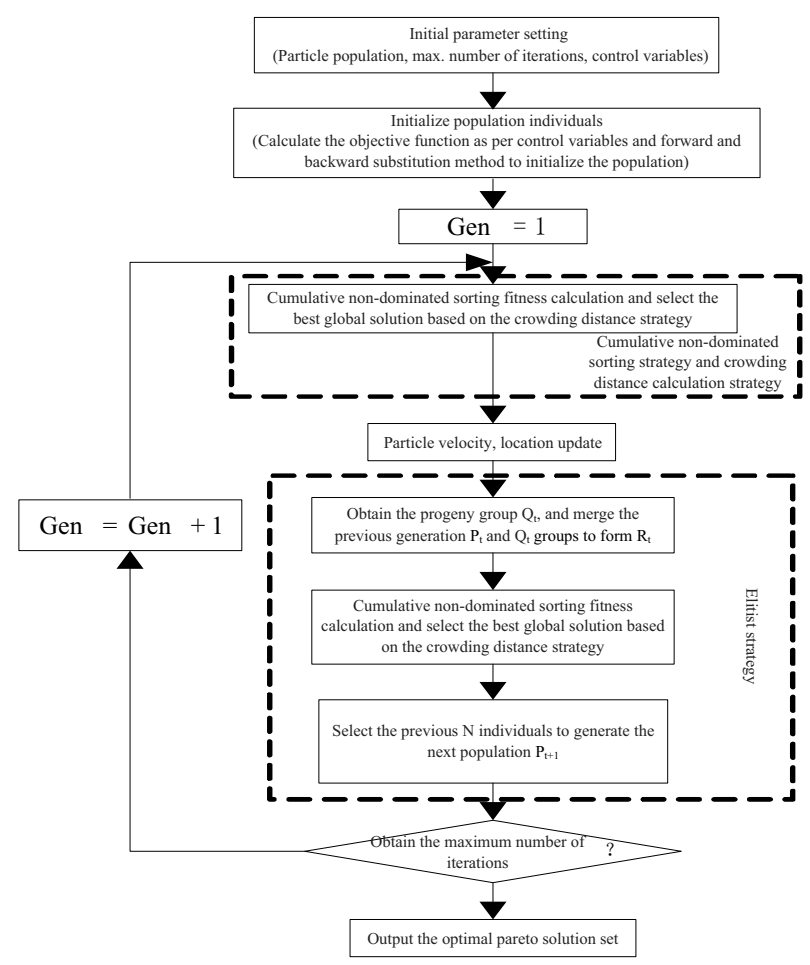

Figure 1. The process of MPSO algorithm 
The strategies of cumulative rank fitness assignment, elitist and crowding distance computation are introduced during the process of calculating, and the flow chart of MPSO as shown in Fig. 1.

\section{Example analisis}

In this paper, the distribution system of IEEE-33 node is chose as an example and the corresponding system structure as shown in Fig. 2. GP including wind power (WT), photovoltaic (PV), fuel cell (FC), miniature turbine (MT), diesel engine (DE) and capacitor group (CB), and the related data of investment and pollution emissions are shown in Table 1. using to the multi-objective mathematical model, and use the MPSO algorithm for optimal allocation of GP, and the initial parameters of MPSO algorithm as follows: initial population of particles is set as 90, the number of iterations is set as 200 , the inertia weight updating interal $[0.45,0.9]$, learning factor $\mathrm{c} 1=\mathrm{c} 2=1$.

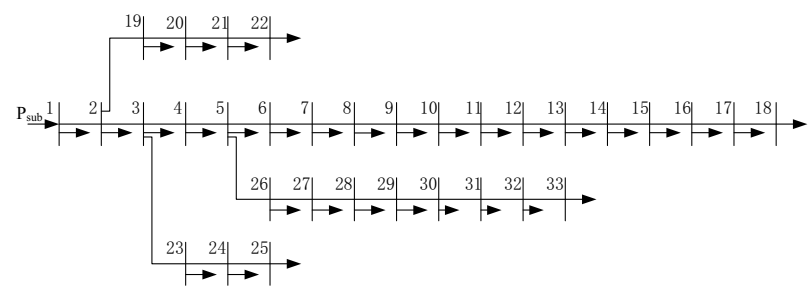

Figure 2. IEEE-33 node distribution network

Table 1. The related data of GP.

\begin{tabular}{|c|c|c|c|c|c|c|}
\hline \multirow{2}{*}{ Genre } & \multirow{2}{*}{$\begin{array}{c}\text { Assets } \\
\text { cost/(\$/kW) }\end{array}$} & \multicolumn{5}{|c|}{$\begin{array}{c}\text { Pollution gas emissions } \\
\text { targets }(\mathrm{kg} / \mathrm{kwh})\end{array}$} \\
\cline { 3 - 7 } & & $\mathrm{CO}_{2}$ & $\mathrm{N0}_{\mathrm{x}}$ & $\mathrm{SO}_{2}$ & $\mathrm{CO}$ & $\mathrm{PM}_{10}$ \\
\hline $\mathrm{WT}$ & 4500 & 0 & 0 & 0 & 0 & 0 \\
\hline PV & 5000 & 0 & 0 & 0 & 0 & 0 \\
\hline FC & 3500 & 0.46 & 0.006 & 0.012 & 0.002 & 0 \\
\hline MT & 1500 & 0.72 & 0.091 & 0.002 & 0.247 & 0.018 \\
\hline DE & 500 & 0.65 & 2.483 & 0.093 & 1.275 & 0.16 \\
\hline $\mathrm{CB}$ & $90 \$ / \mathrm{kvar}$ & 0 & 0 & 0 & 0 & 0 \\
\hline
\end{tabular}

\subsection{Optimal allocation of GP considering benefits of investment and voltage stability}

In this section, from the angle of investment operation efficiency and voltage stability to optimize configuration of GP, and the Pareto solutions can be shown in Fig. 3.

From Fig. 3, it's not hard to see that the relationship between the benefits of investment and the index of voltage stability approximation opposite. In other words, to improve the load side voltage stability performance needs at the expense of the operation efficiency of power grid. Different schemes (A, B, C) are selected to analyze in this section, and the results can be shown in Table 2 and Fig. 4.

The Table 2 and Fig. 4 show that the level of voltage distribution is improved and the network loss is decreased with the GP reasonable access to ADN. For the more, optimal configuration of GP can improve the level of penetration of distributed power supply. Compared with un-optimization schemes, the related level of investment benefit, VSI, network loss are improved after optimization. In $\mathrm{A}$, the index of VSI is higher and the efficiency of investment opration is lowest; In C, the index of VSI is the lower and the efficiency of investment opration is the highest; In B, the factors of VSI and investment benefits are considered reasonable, and the related index is more better than scheme $\mathrm{A}$ and scheme $\mathrm{C}$, and the corresponding optimization results can be shown in Fig. 5.

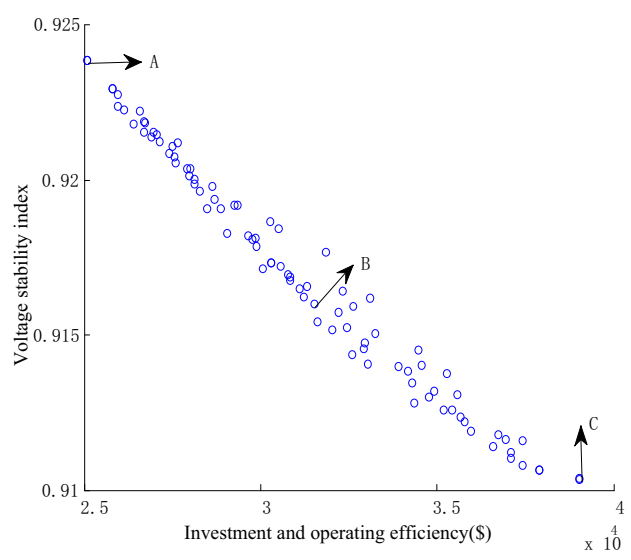

Figure 3. Pareto solution of GP optimization

Table 2. The optimal results of typical schemes.

\begin{tabular}{|c|c|c|c|}
\hline scheme & benefit(\$) & VSI & Powerloss(kW) \\
\hline unoptimized & - & 0.8928 & 202.66 \\
\hline scheme A & 25109 & 0.9239 & 134.19 \\
\hline scheme B & 32060 & 0.9152 & 143.16 \\
\hline scheme C & 39073 & 0.9104 & 148.63 \\
\hline
\end{tabular}

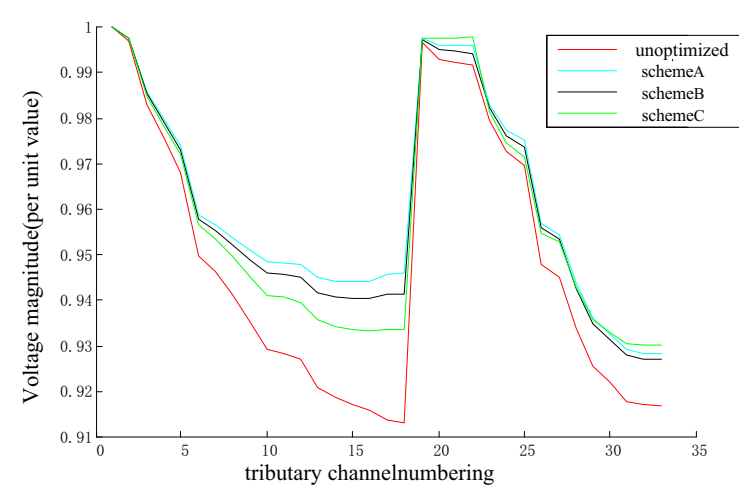

Figure 4. Voltage level under different schemes

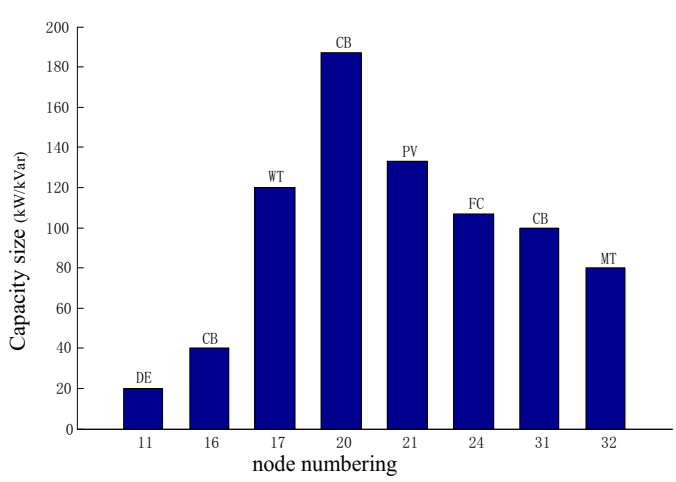

Figure 5. Optimal allocation of GP in scheme B 


\subsection{Optimal allocation of GP considering benefits of investment and pollution gas emissions}

From the angle of investment operation efficiency and the index of pollution gas emission to optimize configuration of GP, and the Pareto solutions can be shown in Fig. 6.

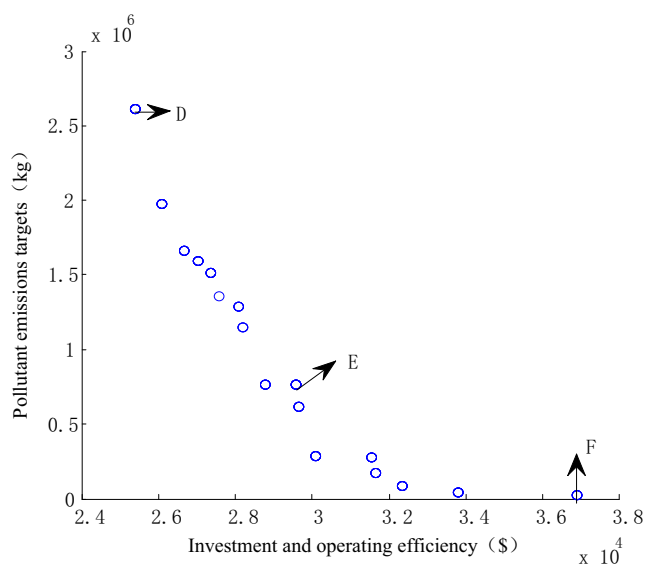

Figure 6. Pareto solution of GP optimization

From the Fig. 6, the benefits of investment is generally inverse-correlated to the index of pollution gas emission, and the reason can be explained as the capacitor groups is considered in the process of optimizing of GP. The low investment cost of capacitor groups and it has no pollution emission, therefore, it's particularly important to consider the influence of capacitor groups in the process of optimizing of GP. Different schemes (D, E, F) are analyzed in this section, and the results can be shown in Table 3 and Fig. 7.

Table 3. The optimal results of typical schemes

\begin{tabular}{|c|c|c|c|}
\hline scheme & $\begin{array}{c}\text { investment } \\
\text { benefit (\$) }\end{array}$ & $\begin{array}{c}\text { gas emission } \\
(\mathrm{kg})\end{array}$ & $\begin{array}{c}\text { power loss } \\
(\mathrm{kW})\end{array}$ \\
\hline Unoptimized & - & - & 202.66 \\
\hline scheme D & 25442 & 2604488 & 117.95 \\
\hline scheme E & 29628 & 760788 & 124.97 \\
\hline scheme F & 36911 & 21024 & 148.75 \\
\hline
\end{tabular}

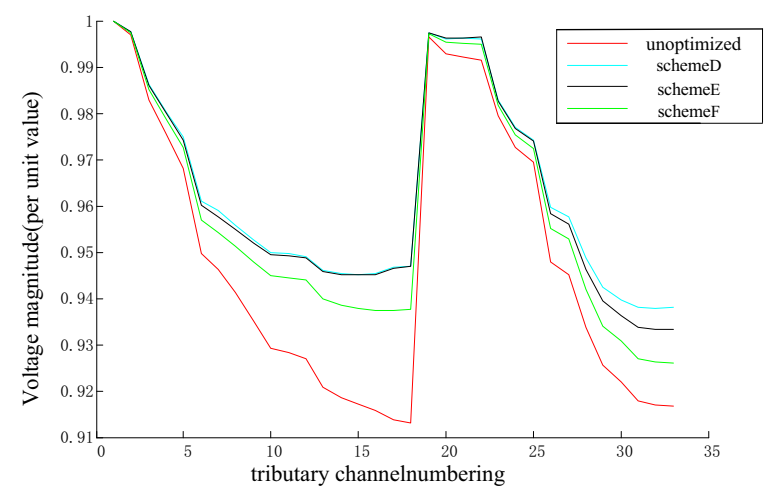

Figure 7. Voltage level under different schemes

From Table 3 and Fig. 7, the index of VSI is higher in scheme D, but, the benefit of investment is the lowest and the emission of pollution gas is the highest. In scheme F, the situation is reversed. In scheme $\mathrm{E}$, the optimal allocation results is reasonablely, and the corresponding results can be shown in Fig. 8 .

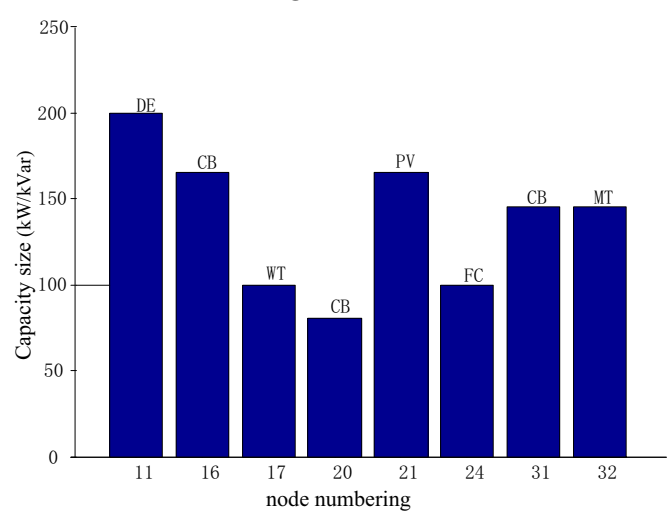

Figure 8. Optimal allocation of GP in scheme E

\subsection{Optimal allocation of GP under different load levels}

In this section, the IEEE-33 node is chose as examples, and the economy, technology and environmental factors are considered in the process of optimizing GP under different load levels $\left(\mu_{1}=0.7, \mu_{2}=1.0, \mu_{3}=1.3\right)$. When $\mu_{2}=1.0$, the pareto solutions of different systems can be shown in Fig. 9. Different evaluation index of optimzing GP can be shown in Table 4 under different conditions.

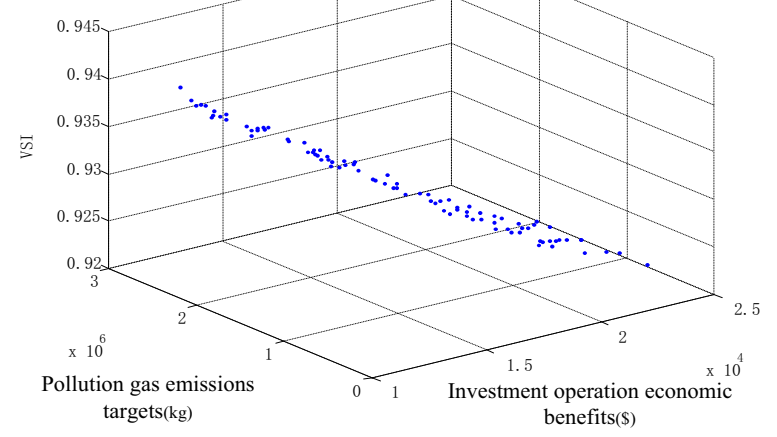

Figure 9. Pareto solution of GP in IEEE-33 node

From Fig. 9, the spatial distribution of pareto solutions is relatively uniform, which shows that the MPSO algorithm has good global search ability. At the same time, the variation patterns of key indexes are basically the same, which reflect that the MPSO algorithm has better parameter identification ability in deal with multi-objective optimization problems.

In Table 4, setting the first schemes as the base under different conditions, and the other two schemes are compared with the first scheme, and the results show that the optimize allocation schemes have obvious difference under different conditions, and the related evaluation index is approximately reversed. The situation of gas emission index is more special is that part of GP have none gas emissions.With the increase of load level, compared with original situation, the efficiency of investment operation and the stability of voltage is improved. On the other hand, compared with different 
optimal allocation schemes with the growth of load level, the capacity of GP is increased, but the stability of voltage is decreased, which explain that the growth trend of existing scale of GP cannot meet the long-term load demand. Therefore, it needs to expand the scale of low carbon perspective of GP.

Table 4. Optimal allocation of GP under different load rate.

\begin{tabular}{|c|c|c|c|c|c|c|c|c|c|c|c|c|}
\hline \multirow[b]{2}{*}{$\mu$} & \multirow[b]{2}{*}{ Plan } & \multicolumn{8}{|c|}{ GP The configuration capacity (Corresponding node location ) } & \multicolumn{3}{|c|}{ assessment criteria } \\
\hline & & $\begin{array}{l}\text { WT } \\
(\mathrm{kW})\end{array}$ & $\begin{array}{c}\mathrm{PV} \\
(\mathrm{kW})\end{array}$ & $\begin{array}{c}\text { FC } \\
(\mathrm{kW})\end{array}$ & $\begin{array}{l}\text { MT } \\
(\mathrm{kW})\end{array}$ & $\begin{array}{c}\mathrm{DE} \\
(\mathrm{kW})\end{array}$ & $\begin{array}{c}\text { CB1 } \\
\text { (Kvar) }\end{array}$ & $\begin{array}{c}\text { CB2 } \\
\text { (Kvar) }\end{array}$ & $\begin{array}{l}\text { CB3 } \\
\text { (Kvar) }\end{array}$ & $\begin{array}{l}\text { benefit } \\
(104 \$)\end{array}$ & VSI & $\begin{array}{l}\text { pollution } \\
\text { gas }(106 \mathrm{Kg})\end{array}$ \\
\hline \multirow{3}{*}{$\mu 1$} & A1 & $165(1)$ & $80(21)$ & $200(24)$ & $180(32)$ & $180(11)$ & $120(20)$ & $120(16)$ & $123(31)$ & 0.4201 & 0.9521 & 2.6508 \\
\hline & A2 & $80(17)$ & $180(21)$ & $200(24)$ & $160(32)$ & $60(11)$ & $160(20)$ & $40(16)$ & $200(31)$ & $0.4717 \uparrow$ & $0.9497 \downarrow$ & $2.9125 \uparrow$ \\
\hline & A3 & 20(17) & 193(21) & $20(24)$ & $150(32)$ & $180(11)$ & $180(20)$ & $130(16)$ & $20(31)$ & $0.9617 \uparrow$ & $0.9391 \downarrow$ & $0.8410 \downarrow$ \\
\hline \multirow{3}{*}{$\mu 2$} & B1 & $126(1)$ & $0(21)$ & $100(24)$ & $140(32)$ & 145(11) & $100(20)$ & $160(16)$ & $60(31)$ & 1.2483 & 0.9381 & 2.6570 \\
\hline & B2 & $180(17)$ & $200(21)$ & $152(24)$ & $40(32)$ & 180(11) & $100(20)$ & $120(16)$ & $180(31)$ & $1.3012 \uparrow$ & $0.9364 \downarrow$ & $2.9220 \uparrow$ \\
\hline & B3 & 161(17) & $80(21)$ & $60(24)$ & $0(32)$ & $80(11)$ & $20(20)$ & $40(16)$ & $120(31)$ & $2.0718 \uparrow$ & $0.9256 \downarrow$ & $1.7639 \downarrow$ \\
\hline \multirow{3}{*}{$\mu 3$} & $\mathrm{C} 1$ & $120(17)$ & $160(21)$ & $20(24)$ & $140(32)$ & $157(11)$ & $180(20)$ & $120(16)$ & $180(31)$ & 2.4968 & 0.9239 & 2.8842 \\
\hline & $\mathrm{C} 2$ & $160(17)$ & $186(21)$ & $140(24)$ & $117(32)$ & $160(11)$ & $106(20)$ & $175(16)$ & $132(31)$ & $2.5595 \uparrow$ & $0.9228 \downarrow$ & $2.9220 \uparrow$ \\
\hline & $\mathrm{C} 3$ & $104(17)$ & $20(21)$ & $190(24)$ & $60(32)$ & $40(11)$ & $60(20)$ & $150(16)$ & $20(31)$ & $3.8707 \uparrow$ & $0.9104 \downarrow$ & $1.3553 \downarrow$ \\
\hline
\end{tabular}

\section{Conclusion}

(1) A simple and effective index of voltage stability is put forward to assess the impact on voltage quality with GP access to DN. Rational allocation of GP can effectively improve the operation level of DN.

(2) A multi-objective optimition algorithm is proposed to optimize allocation of GP in DN, and this algorithm has better optimization performance and efficiency.

(3) With the increase of load level, rational planning of GP can improve the economic benefit for power supply service, and improve the quality of voltage for power users, and can also provide auxiliary comprehensive and reasonable decision for power dispatchers.

\section{References}

1. Y. Zhao, Y. An, Q. Ai. Research on size and location of distributed generation with vulnerable node identification in the active distribution network. IET
Generation, Transmission \& Distribution, 8(11): 1801-1809 (2014)

2. C.L. T. Borges, V.F. Martins. Multistage expansion planning for active distribution networks under demand and distributed generation uncertainties. Journal of Electrical Power \& Energy Systems, 36(1): 107-116 (2012)

3. L. Li, W. Tang, M.K. Bai. Multi-objective locating and sizing of distribution generators based on timesequence characteristics. Automation of Electric Power Systems, 37(3): 58-64 (2013)

4. Z. Wang, B. Chen, J. Wang, et al. Robust optimization based optimal DG placement in microgrids. IEEE Transactions on Smart Grid, 5(5): 2173-2182 (2014)

5. D. Foster, M. Berry, N. Boland, et al. Comparison of mixed-integer programming and genetic algorithm methods for distributed generation planning. IEEE Transaction on Power System, 29(2): 833-843 (2014)

6. D.Q. Hung, N. Mithulananthan, K.Y. Lee. Determining PV penetration for distribution systems with time-varying load models. IEEE Transaction on Power System, 29(6): 3048-3057 (2014) 